\title{
Was kann die Rehabilitation onkologischer Patienten nach kurativer und palliativer Therapie leisten?
}

\author{
H.H. Bartsch \\ Klinik für Onkologische Rehabilitation und Nachsorge, Klinik für Tumorbiologie an der Albert-Ludwigs Universität Freiburg
}

Ähnlich dem gesellschaftlichen und ökonomischen Wandel, ist auch unser Gesundheitssystem in einen permanenten Prozess der inhaltlichen und strukturellen Veränderung einbezogen. Beide Merkmale werden in erheblichem Maß von gesundheitspolitischen Überlegungen geprägt. Eine der größten Herausforderungen unserer Zeit mit entsprechend gravierenden Auswirkungen auf alle Bereiche des Gesundheitssystems dürfte die bevorstehende Einführung der DRG (diagnosis-related group)-Systeme sein. Diese betreffen zunächst nur den akutmedizinischen, stationären Behandlungsbereich, haben aber zwangsläufig direkte Auswirkung auch auf andere Leistungsbereiche wie den der stationären und ambulanten medizinischen Rehabilitation. Dies bedeutet, dass die bisherigen Modelle der Kooperation zwischen akutmedizinischem und rehabilitativem Bereich neu strukturiert werden müssen. Für die Onkologie stellen sich dabei erneut die Fragen, zu welchem Zeitpunkt und mit welchen Inhalten rehabilitationsonkologische Maßnahmen sinnvoll zur Optimierung der Patientenversorgung eingebunden werden können.

Die demographische Entwicklung der Altersstruktur in den westeuropäischen Ländern und die alterskorrelierte Krebsprävalenz lässt befürchten, dass sich in den nächsten Jahren die Anzahl der Krebspatienten verdoppeln wird. In diesem Zeitraum ist damit zu rechnen, dass Krebserkrankungen zur Todesursache Nummer eins werden. Aufgrund der Inzidenzzahlen und mittleren Überlebenszeiten der wichtigsten und häufigsten Krebserkrankungen kann davon ausgegangen werden, dass beispielsweise in der Bundesrepublik Deutschland schon derzeit zirka 1 Million Personen tumorkrank sind. Zum Zeitpunkt der Diagnosestellung sind jedoch nur etwa 50\% der Patienten kurativ behandelbar. Die in den vergangenen Jahrzehnten intensiv propagierten Krebs-Früherkennungsuntersuchungen sowie die Fülle neuer Therapieoptionen haben an diesem Umstand nur wenig geändert. Daraus ergibt sich die Tatsache, dass es neben den kurativ behandelten Tumorpa- tienten, die zum Teil mit erheblichen Therapiefolgestörungen leben müssen, eine große Anzahl von Patienten in palliativen Behandlungssituationen gibt. Diese sind sowohl durch die zugrundeliegende Tumorerkrankung als auch durch mehr oder weniger ausgeprägte Behandlungsfolgen in ihren körperlichen wie psychosozialen Funktionen beeinträchtigt. Für beide Patientengruppen besteht ein grundsätzlicher Anspruch auf rehabilitative Versorgung. Vor dem Hintergrund der dargestellten Strukturveränderungen im Gesundheitswesen ist jedoch eine heftige Diskussion über die Punkte Rehabilitationsfähigkeit, Rehabilitationsbedürftigkeit und Fortführung (akut-) medizinischer Maßnahmen gerade auch bei onkologischen Patienten $\mathrm{zu}$ erwarten. Insofern stehen Fragen nach der Effektivität und Effizienz, aber auch der Kostenerstattung rehabilitativer Therapien für kurativ und palliativ versorgte Tumorpatienten wieder im Vordergrund.

\section{Rehabilitation soll Defizite verringern}

Rehabilitation ist die Behandlung Kranker, Genesender oder Behinderter mit speziellen Mitteln und Maßnahmen, um vorhandene oder absehbare gesundheitliche Schädigungen zu verringern und Restfunktionen zu verbessern. Neben der Verbesserung der gesundheitlichen Situation soll eine dauerhafte Integration der Patienten in Familie, Gesellschaft, Arbeit und Beruf ermöglicht werden. Die interdisziplinär umgesetzte Rehabilitation onkologischer Patienten nimmt in Deutschland insofern eine Sonderstellung ein, da die erforderlichen Maßnahmen im überwiegenden Fall von den Rentenversicherungsträgern finanziert werden. Dies gilt nicht nur für berufstätige Personen, sondern auch gleichermaßen für Empfänger von Altersruhegeld. Ziel soll in jedem Fall die Verbesserung der gesundheitlichen Situation des einzelnen Patienten sein. Damit lassen sich die Rehabilitationsziele am besten mit folgenden Stichworten charakterisieren:

\begin{tabular}{ll}
\hline KARGER & $\odot$ 2002 S. Karger GmbH, Freiburg \\
Fax +497614520714 & Accessible online at: \\
$\begin{array}{l}\text { E-mail Information@Karger.de } \\
\text { www.karger.com }\end{array}$ & www.karger.com/journals/onk
\end{tabular}


Tab. 2. Psychosoziale Problemfelder bei onkologischen Patienten

\begin{tabular}{|c|c|}
\hline Bereich & Problem \\
\hline Emotionale Probleme & $\begin{array}{l}\text { Angst } \\
\text { Depression } \\
\text { Verunsicherung } \\
\text { Regression } \\
\text { Erschöpfung }\end{array}$ \\
\hline Somatisierungsstörungen & $\begin{array}{l}\text { Inappetenz, Übelkeit und Erbrechen } \\
\text { Gewichtsverlust } \\
\text { Diarrhö } \\
\text { Schlafstörungen } \\
\text { Compliance }\end{array}$ \\
\hline Neuropsychologische Defizite & $\begin{array}{l}\text { Konzentrationsausdauer } \\
\text { Gedächtnis }\end{array}$ \\
\hline Soziale, familiäreProbleme & $\begin{array}{l}\text { Krankheits- und therapie assoziierte } \\
\text { Einflüsse auf das soziale Netz } \\
\text { Sexualität } \\
\text { Kommunikation in Partnerschaft }\end{array}$ \\
\hline Berufliche Veränderung & $\begin{array}{l}\text { Änderung der Einkommenssituation } \\
\text { Anpassung der beruflichen Situation }\end{array}$ \\
\hline
\end{tabular}

z.B. eine erforderliche Schmerztherapie mit Opiaten, haben mitunter eine erhebliche Leistungseinschränkung im körperlichen und kognitiv-emotionalen Bereich zur Folge.

Weiterhin bestehen enge Verbindungen zwischen dem Symptomkomplex Fatigue und Depressionen bei Tumorpatienten. Auch wenn depressive Störungen in ihrer Prävalenz mit zirka $20 \%$ bei Tumorpatienten nicht häufiger nachzuweisen sind als bei Patienten mit anderen chronischen Erkrankungen, müssen sie im Zusammenhang mit der Untersuchung von Fatigue miterfasst werden.

In der Ätiologie psychosozialer Probleme stehen die als existentiell bedrohlich empfundene Grunderkrankung mit der oft nur schwer abzuschätzenden Perspektive sowie die Belastungen durch die Tumortherapiemaßnahmen im Vordergrund. In Tabelle 2 sind verschiedene spezifische psychosoziale Problembereiche für onkologische Patienten zusammengefasst.

Im Hinblick auf eine psychosoziale Rehabilitationsbedürftigkeit spielen natürlich sowohl die vorbestehenden Persönlichkeitsmerkmale des Patienten als auch der Umfang der Unterstützung im familiären oder sonstigen sozialen Umfeld eine Rolle. Dabei besteht oft eine Situation, die - neben depressiven und zum Teil angstneurotischen Zustandsbildern - am ehesten gekennzeichnet ist durch ein wechselndes Ausmaß an Antriebslosigkeit und psychischer Erschöpfung. Erst in den letzten Jahren deutlicher identifizierbar sind spezifische kognitive, neuropsychologische und intellektuelle Defizite im Sinne von Konzentrationsstörungen sowie Einschränkungen der Merk- und Gedächtnisfähigkeit. Bei diesen Störungen sind vorangegangene Tumortherapien (Chemotherapie, Radiatio) als wesentliche Mitverursacher anzusehen. Nicht selten
Tab. 3. Ziele der Rehabilitation in kurativer bzw. palliativer Erkrankungssituation

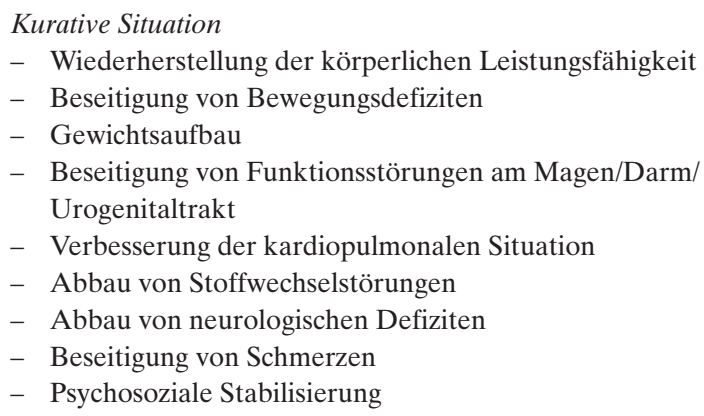

- Wiederherstellung der körperlichen Leistungsfähigkeit

- Beseitigung von Bewegungsdefiziten

- Gewichtsaufbau

- Beseitigung von Funktionsstörungen am Magen/Darm/ Urogenitaltrakt

- Verbesserung der kardiopulmonalen Situation

- Abbau von Stoffwechselstörungen

- Abbau von neurologischen Defiziten

- Beseitigung von Schmerzen

- Psychosoziale Stabilisierung

\section{Palliative Situation}

- Verbesserung der körperlichen Leistungsfähigkeit

- Adaptation an Organfunktionsstörungen

- Hilfsmittelversorgung (Stoma, Gehhilfe usw.)

- Optimierung der Schmerztherapie

- Psychosoziale Unterstützung

- Erhalt der Selbständigkeit / Verhinderung von Pflegebedürftigkeit

bestehen bei lange andauernder Erkrankung und entsprechenden Aufenthalten in Kliniken soziale Rückzugstendenzen, die auch eine konsequente Einbindung in ein rehabilitatives Programm erheblich beeinträchtigen können. Auch besteht häufig eine große Unsicherheit und Hilflosigkeit der direkten Angehörigen bzw. Freunde im Umgang mit den Erkrankten.

\section{Rehabilitationsziele in kurativen Behandlungs- situationen}

Die Rehabilitationsziele müssen in Abhängigkeit vom Zeitpunkt der rehabilitativen Maßnahme, vom Ausmaß der Grunderkrankung und deren tumortherapeutischer Strategie, von Begleiterkrankungen und Problemen sowie weiterer Einflussfaktoren für jeden Patienten nach einem individuellen «Assessment» definiert werden. Im Vordergrund stehen bei den somatischen Zielen die möglichst rasche und weitgehende Wiederherstellung der körperlichen Leistungsfähigkeit, die Minderung krankheits- und/oder therapiebedingter Störungen, die möglichst optimale Adaptation an dauerhafte Beeinträchtigungen, die nicht nur pharmakologische Beeinflussung von Schmerzen und der Versuch, eine drohende Unselbständigkeit zu verhindern. Im psychosozialen/emotionalen Bereich sind Strategien der Krankheitsauseinandersetzung und -verarbeitung sowie die Einflussnahme auf die oft zahlreichen somatisierten Störungen und die Hilfestellung zur Überwindung der sozialen Isolation zu nennen. Weiterhin kann eine Verbesserung der kognitiven Leistungsfähigkeit, die gemeinsam mit den somatischen Einschränkungen unter Umständen zu einem erheblichen Defizitgefühl der Patienten beiträgt, erreicht werden. Im sozialmedizinischen Bereich ist das Ziel der 
Rehabilitation - nach einer sorgfältigen Analyse der bisherigen beruflichen Anforderungen - eine in Ausmaß und Geschwindigkeit der Leistungsfähigkeit des Patienten angepasste berufliche Wiedereingliederung (Tab. 3).

Als weiteres wesentliches Ziel onkologischer Rehabilitationsstrategien ist die ausführliche Information und Beratung der Patienten anzuführen. Dabei geht es einerseits um sehr krankheitsspezifische und tumortherapeutische Themen, aber ebenso um verhaltenstherapeutische Ansätze im Sinne von Coping-Strategien.

\section{Rehabilitationsziele in palliativen Krankheitssituationen}

Für Patienten in palliativen Krankheitssituationen hat die Verbesserung der körperlichen Leistungsfähigkeit einen ebenso großen Stellenwert wie für Patienten nach kurativer Tumortherapie. Allerdings ist die Perspektive für den Patienten, seine «Normalität» wieder $\mathrm{zu}$ erreichen, deutlich eingeschränkter. In diesem Fall ist enorme Motivationsarbeit durch das Therapeutenteam zu leisten, um vorhandene Ressourcen zu nutzen. Ähnlich gilt dies auch für die Adaptation an funktionelle Defizite, z.B. im Gastrointestinal- oder Urogenitaltrakt, insbesondere vor der Perspektive drohender Verschlechterung.

Eine grundsätzliche Testierung der Erwerbsunfähigkeit bedingt durch eine palliative Erkrankungssituation sollte ohne ausführliche Diskussion mit dem Patienten über seine Wünsche und Vorstellungen auf keinen Fall erfolgen. Gerade die Berufstätigkeit, in der Regel zeitlich reduziert bzw. mit einem hohen Maß an Flexibilität, kann für viele Tumorpatienten noch für längere Zeit das entscheidende Stück «Normalität» im Alltag mit entsprechender sozialer Anbindung darstellen. Die Entwicklung eines tragfähigen psychosozialen Unterstützungskonzepts unter Einbeziehung der Angehörigen hat oberste Priorität für Patienten in palliativen Erkrankungssituationen.

\section{Rehabilitationsmaßnahmen}

\section{Therapie}

Korrespondierend zu den oben genannten Rehabilitationsbedürfnissen und Rehabilitationszielen, erstrecken sich die therapeutischen Strategien auf eine ganze Reihe somatischer und psychosozialer Problemfelder. Am Anfang der Therapieplanung steht eine ausführliche Bestandsaufnahme der individuellen körperlichen Situation. Neben den medizinischen Rahmenbedingungen wie spezielle Probleme durch eingeschränkte Organfunktionen, Schmerzsymptomatik oder Appetitlosigkeit spielt die Einschätzung der körperlichen Leistungsfähigkeit durch qualifizierte Physiotherapeuten eine wesentliche Rolle. Die Selbsteinschätzung der Patienten sowie der bisherige Stellenwert ihrer körperlichen Aktivitäten muss in die Analyse mit einbezogen werden. Das Therapieprogramm soll ausgewogen sein und sich sowohl im Hinblick auf Inhalt und Intensität an den Fähigkeiten und Wünschen im Sinne der oben genannten «individuellen Normleistung» des Patienten orientieren. Die Notwendigkeit von Einzelbehandlungen ist bei Patienten in palliativen Erkrankungssituationen gegenüber denen in kurativen Situationen in der Regel erhöht. Das körperliche Training mit speziellen krankengymnastischen Einzelbehandlungen und zum Teil vorsichtig dosierten Versuchen zur Steigerung der Ausdauerleistung, z.B. auf dem Laufband oder auch Fahrradergometer, kann durch ein Gehtraining ergänzt werden.

Die in unserer Klinik umgesetzte Strategie in der Schmerzbehandlung schließt neben rein medikamentösen Maßnahmen je nach Ausgangssituation und verbliebenen Restfunktionen durchaus physiotherapeutische Ansätze wie auch psychosoziale Therapien mit ein.

Im Mittelpunkt der psychosozialen Maßnahmen für die $\mathrm{Pa}-$ tienten stehen die Unterstützung in der Auseinandersetzung mit der Erkrankungssituation durch Stärkung der eigenen Kompetenz, die Entwicklung von Perspektiven und deren Umsetzungsmöglichkeiten, der Abbau von zum Teil erheblichen Belastungsfaktoren und die Planung der weitergehenden Betreuung. Anlässlich des Erstkontakts mit einem psychosozialen Mitarbeiter wird, vergleichbar zu dem somatischen «Assessment», sowohl die Selbsteinschätzung wie auch die Fremdbeurteilung als Planungsbasis für psychologisch-kreativtherapeutische Maßnahmen benutzt. Dabei werden insbesondere Bereiche wie emotionale Belastung, neuropsychologisch-kognitive Einschränkungen, familiäre Belastungen und körperliche Einschränkungen berücksichtigt. Zum Teil können standardisierte Erfassungsinstrumente (z.B. Lebensqualität-Fragebogen, neuropsychologische Evaluation, Messinstrumente für Angst/Depression) eingesetzt werden. An therapeutischen Möglichkeiten stehen Einzelbetreuung, Gruppenbetreuung, Entspannungsübungen, themenspezifische Gesprächsgruppen und Gesprächskreise zur Verbesserung sozialer Kontakte zur Verfügung.

Wesentlich bei allen therapeutischen Maßnahmen sowohl des somatischen wie psychosozialen Bereichs ist die intensive interdisziplinäre Abstimmung, um einerseits optimal die noch vorhandenen Ressourcen der Patienten zu nutzen und zum anderen entsprechend der individuellen Bedürftigkeit inhaltlich und zeitlich abgestimmte Schwerpunkte setzen zu können.

\section{Information/Motivation/Schulung}

Der Informationsbedarf von onkologischen Patienten ist generell außerordentlich hoch. Im Vordergrund stehen verständlicherweise Fragen zur Erkrankung und zu möglichen Therapieoptionen sowie deren Auswirkungen auf das körperliche Befinden und die allgemeine Lebensqualität. Darüber hinaus besitzen aber auch Aspekte der Rezidivverhütung und damit auch eigener Steuerungsmöglichkeiten dieses Risikos 
einen hohen Stellenwert. Dies beinhaltet Informationen zur Ernährung, zu sogenannten unkonventionellen Mitteln in der Krebsmedizin (UMK) wie auch zu Möglichkeiten struktureller und ökonomischer Hilfe durch unser Sozialversicherungssystem. Außerdem werden psychosoziale Auswirkungen auf Partnerschaft, Familie, Freundeskreis und Berufsleben thematisiert sowie Empfehlungen zum Verhalten zu Hause und außer Haus gegeben. Im Rahmen intensiver Gespräche müssen einerseits Informationen zu den jeweiligen Grunderkrankungen, zu deren weiterem eingeschätzten Verlauf und zur voraussichtlichen Beeinflussbarkeit durch eine Behandlung vermittelt werden. Neben der damit grundsätzlich möglichen Verbesserung der Krankheitsverarbeitung soll dies auch dem Abbau erheblicher Zweifel und eines damit möglicherweise einhergehenden übertriebenen Diagnostikbedürfnisses dienen. Die sowohl nach kurativer Tumortherapie, aber mehr noch in einer fortgeschrittenen Krankheitssituation gegebenen zahlreichen Empfehlungen aus dem Freundes- und Bekanntenkreis über neue Diagnostik/Therapiemöglichkeiten lassen immer wieder Zweifel und Hoffnung aufkeimen, die mitunter mehr Verunsicherung bewirken, als dem Auseinandersetzungsprozess mit der Erkrankung dienlich ist.

Ein klassischer Bereich, in dem sich diese Problematik widerspiegelt, sind Fragen zur Ernährung. Einerseits bestehen oft gravierende Einschränkungen des Appetits, zum Teil aber auch funktionelle Störungen im Digestionstrakt; andererseits setzen sich die Patienten mit abstrusen Diätvorschlägen und zahllosen Supplementierungsvorschlägen auseinander. Hier kann nur eine sehr individuelle Ernährungsberatung auf der Grundlage der Wünsche des Patienten, seiner Gesamtverfassung und seiner vorgegebenen Funktionsbeeinträchtigungen des Verdauungstrakts erfolgen. Der Beratung der jeweiligen Partner, von deren Seite gerade in fortgeschrittenen Erkrankungssituationen oft ein nicht unerheblicher Druck auf die Patienten ausgeübt wird, kommt dabei eine ebenso große Bedeutung zu.

\section{Qualitätsmanagement}

Die Praxis der medizinischen Rehabilitation onkologischer Patienten hat sich in den vergangenen 20 Jahren erheblich verändert. Dieser Entwicklungsprozess wurde und wird nur sehr bedingt seitens der Akutmediziner wahrgenommen. Einer der Gründe mag in der nur sehr begrenzt durchgeführten wissenschaftlichen Bearbeitung rehabilitativer Fragestellungen in der Onkologie liegen. Ebenso ist nach wie vor in vielen Köpfen die Überzeugung, dass es ein «Qualitätsgefälle» zwischen Akut- und Rehabilitationsmedizin gibt, fest verankert.

Mit Einführung des Qualitätssicherungsprogramms der gesetzlichen Rentenversicherung für den Bereich der medizinischen Rehabilitation wurden jedoch wesentliche Voraussetzungen zur Beurteilung der klinikübergreifenden und trägerübergreifenden Prozessqualität geschaffen. Dabei stellt das «Peer review»-Verfahren das Kernstück zur Analyse der Prozessqualität dar. Die formulierten Anforderungen an dieses
Verfahren waren 1. Aufdecken von Schwachstellen im Rehabilitationsprozess, 2. Möglichkeit des Klinikvergleichs bezüglich der Prozessqualität und 3. hohe Praktikabilität.

Eine Checkliste mit 52 qualitätsrelevanten Merkmalen, mit denen Stichproben von Entlassungsberichten analysiert werden, wurde entwickelt. Weiterhin wird die Prozessqualität im Rahmen einer Befragung zur Patientenzufriedenheit erhoben. Eine derartige Systematik, wie sie seitens der Rentenversicherungen eingeführt wurde, ist im akutmedizinischen Bereich noch nicht zu erkennen.

\section{Neue Rehabilitationsschwerpunkte und -forschung in der Onkologie}

Die Entwicklungen innerhalb der Hämatologie und Onkologie haben auch neue Fragen und einen neuen Bedarf im Hinblick auf rehabilitative Ansätze aufgeworfen. So hat z.B. die Zahl hämatologischer Stammzelltransplantationen (HSCT) in den letzten Jahren, parallel zum internationalen Trend, auch in Deutschland deutlich zugenommen. Die Intensität dieser Intervention hat für die betroffenen Patienten neben zahlreichen akuten Risiken auch eine Fülle verzögert auftretender Störungen zur Folge, die einen potentiell hohen Rehabilitationsbedarf induzieren [9].

Als Beispiel eines in den letzten Jahren neu entwickelten Rehabilitationsangebots sei stellvertretend das Konzept der Rehabilitation von Patienten nach allogener Stammzelltransplantation erwähnt. Durch eine enge Kooperation zwischen primär transplantierenden Abteilungen und ausgesuchten onkologischen Rehabilitationsfachkliniken konnte für diese problematische Patientengruppe mit hohem Rehabilitationsbedarf eine erhebliche Verbesserung der posttransplantären Versorgungssituation erreicht werden. Inzwischen sind klare Standards und Qualitätsanforderungen formuliert, deren Umsetzung unabdingbare Voraussetzung zur Betreuung dieser Patientengruppe ist [10]. Dies gilt ganz besonders für Patienten, die sich in der noch risikoreichen direkten Posttransplantationsphase befinden. Bei der Rehabilitation nach hämatologischer Stammzelltransplantation wurde ebenfalls deutlich, dass die gegenseitige Anerkennung der Betreuungskompetenz für bestimmte Erkrankungs- und Behandlungsphasen auf Seiten der Akut- und der Rehabilitationsmedizin letztlich zum Nutzen der Patienten führt. Insofern müssen die Überlegungen vieler Primärkrankenhäuser, den Zwang des Bettenabbaus durch die Einrichtung kleinerer Rehabilitationsabteilungen zu kompensieren, äußerst kritisch hinterfragt werden. Es wäre tragisch, wenn aufgrund derartiger Umstrukturierungen ein Versorgungsbereich mit jetzt noch hoher Struktur- und Prozessqualität zum verlängerten, dafür etwas preiswerteren Arm akutmedizinischer Versorgung degenerieren würde.

Die Rehabilitationsforschung allgemein und entsprechend auch der Teilaspekt onkologischer Rehabilitationsforschung haben im Vergleich zu anderen wissenschaftlichen Disziplinen 
noch eine eher kurze Historie. Haaf und Schliehe [11] diskutieren in einem neueren Beitrag den Stand und Bedarf der Rehabilitationsforschung allgemein. Aus gemeinsamen Arbeitskreisen zwischen dem Verband Deutscher Rentenversicherungsträger (VDR) und rehabilitationswissenschaftlichen Forschungsabteilungen resultierte letztlich die Empfehlung einer Förderinitiative zur Verbundforschung Rehabilitationswissenschaften, die 1996 öffentlich ausgeschrieben wurde und seit 1998 insgesamt 85 Projekte fördert. Die Rehabilitationsonkologie war darin bisher lediglich mit vier Einzelprojekten vertreten.

Für den Bereich der onkologischen Rehabilitation besitzt der Aspekt der Erfolgsbeurteilung gegenüber anderen Indikationen oder definierten organischen Funktionsdefiziten eine besondere Bedeutung, da es sich um ein extrem komplexes Gebilde aus einerseits funktionalen Einschränkungen, aber auch der Bearbeitung von Rezidivangst, Selbstwert- und Partnerproblemen, Depressionen und einer sozialen Rückzugsproblematik handelt. Am Beispiel des Mammakarzinoms wurde daher von unserer Arbeitsgruppe seit 1998 eine multizentrische Studie zur zielorientierten Evaluation rehabilitativer Maßnahmen im Sinne eines Vergleichsgruppendesigns durchgeführt [12]. Die Daten befinden sich derzeit in der Auswertung und werden in diesem Jahr publiziert.

Weitere Forschungsthemen umfassen die Problematik neuropsychologischer Defizite nach Tumorerkrankungen und -therapien sowie die Inzidenz und Ausprägung der Fatigue-Symptomatik bei Tumorpatienten.

Diese Beispiele sollen nur verdeutlichen, dass es auch im Anschluss an die akutonkologische Versorgung von Patienten zahlreiche Problemstellungen gibt, die einer soliden wissenschaftlichen Bearbeitung bedürfen. Im Gegensatz zur Akutmedizin und anderen Indikationsbereichen in der Rehabilitationsmedizin (z.B. Rheumatologie/Orthopädie, Neurologie) existiert jedoch bisher keine explizite universitäre Einrichtung, die sich diesen onkologischen Themenbereichen systematisch annehmen kann. Hier besteht ein dringender Handlungsbedarf.

\section{Perspektiven}

Die Rehabilitation onkologischer Patienten hat in den vergangenen 15 Jahren deutlich an Profil gewonnen. Durch Umsetzung eines biopsychosozialen Betreuungsansatzes konnte für zahlreiche somatische und psychosoziale Folgestörungen nach einer Primärtumortherapie, aber auch für Patienten in palliativen Erkrankungssituationen ein nachhaltiger Nutzen erreicht werden. Der bevorstehende Strukturwandel in unserem Gesundheitssystem fordert jedoch dringend neue Kooperationsmodelle und Vereinbarungen zur sinnvollen Einbindung rehabilitativer Maßnahmen in die Versorgungskette. Die sicher eintretende Verkürzung der Liegezeiten in den Akutkliniken nach Einführung der DRGs darf nicht zu Lasten der Rehabilitationskliniken gehen. Eine neue Definition der Rehabilitationsbedürftigkeit und Rehabilitationsfähigkeit darf sich nicht an den organisatorischen Zwängen des akutmedizinischen Bereichs orientieren. Hier bedarf es eines intensiven und fairen Dialogs zwischen den beteiligten Leistungsbereichen.

Der Forschungs- und Weiterbildungsbedarf auf dem Gebiet der onkologischen Rehabilitation ist groß.

Allerdings befindet sich gerade die onkologische Rehabilitation in einem besonderen Spannungsfeld zwischen Bedürfnissen und Anforderungen auf Seiten der Patienten und andererseits den Zuständigkeiten auf Seiten der Kostenträger. Die vorrangig für Rehabilitation verantwortlichen Rentenversicherungen sehen in einer Versichertengruppe mit nur noch teilweise vorhandener Erwerbsperspektive keine eigentliche Zuständigkeit. Dieses Phänomen spiegelt sich unter anderem auch in der niedrigen Bewilligungsquote rehabilitationsonkologischer Forschungsprojekte im Rahmen der Verbundforschung wider.

Es bleibt zu hoffen, dass die Aktivitäten und Möglichkeiten der onkologischen Rehabilitationsmedizin in Zukunft noch besser integriert werden und deren wissenschaftliche Etablierung auch durch die universitären Gremien unterstützt wird.

\section{Literatur}

1 Heim ME: Rehabilitation in der integrativen Behandlung von Krebskranken. Onkologie 2001;24 (suppl 5):16-20.

2 Egner U, Gerwinn H, Müller-Fahrnow W, Schliehe F: Das Qualitätssicherungsprogramm der gesetzlichen Rentenversicherung für den Bereich der medizinischen Rehabilitation. Konzept, Stand der Umsetzung und Perspektiven. Rehabilitation 1998; 37:S2-S7.

3 Schmid L, Delbrück H, Bartsch HH, Kruck P: Zur Strukturqualität in der onkologischen Rehabilitation. Rehabilitation 2000;39:350-354.

4 Bartsch HH, Delbrück H, Kruck P, Schmid L: Zur Prozessqualität in der onkologischen Rehabilitation. Rehabilitation 2000;39:355-358.

5 Delbrück H, Schmid L, Bartsch HH, Kruck P: Zur Ergebnisqualität in der onkologischen Rehabilitation. Rehabilitation 2000;39:359-362.
6 van Harten WH, van Noort O, Warmerdam R, Hendricks H, Seidel E: Assessment of rehabilitation needs in cancer patients. Int J Rehabil Res 1998;21:247-257.

7 Schröck R, Schmid L, Sauer H: Rehabilitation von Patientinnen mit Mammakarzinom. Onkologe 2000;6:15-27.

8 Weis J, Bartsch HH (Hrsg): Fatigue bei Tumorpatienten. Eine neue Herausforderung für Therapie und Rehabilitation. Basel, Karger, 2000.

9 Bartsch HH, Finke J, Mumm A (Hrsg): Hämatopoetische Stammzelltransplantation. Neue Konzepte in der Rehabilitation und Nachsorge transplantierter Patienten. Basel, Karger, 2001.
10 Bartsch HH, Mumm A, Delbrück H, Orth HB: Rehabilitation von Patienten nach allogener hämatologischer Stammzelltransplantation, Standards und Qualitätskriterien. Onkologe 2000;6:44-51.

11 Haaf HG, Schliehe F: Zur Situation in der Rehabilitationsforschung: Stand und Bedarf; in Bengel J, Koch U (Hrsg): Grundlagen der Rehabilitationswissenschaften. Heidelberg, Springer, 2000, pp 1939.

12 Weis J, Moser MT, Fachinger D, Bartsch HH: Zielorientierte Evaluation von Maßnahmen der stationären onkologischen Rehabilitation - Multizenterstudie ZESOR; in Bengel J, Jäckel WH (Hrsg): Zielorientierung in der Rehabilitation. Regensburg, Roderer, 2000, pp 177-188. 